\title{
Simplifying amplitudes in Maxwell-Einstein and Yang-Mills-Einstein supergravities
}

\author{
Marco Chiodaroli \\ Max-Planck-Institut für Gravitationsphysik \\ Albert-Einstein-Institut, Am Mühlenberg 1, 14476 Potsdam, Germany \\ marco.chiodaroli@aei.mpg.de
}

\begin{abstract}
This article reviews recent progress in formulating double-copy constructions for scattering amplitudes in supergravity theories with $\mathcal{N}=2$ supersymmetry in five and four spacetime dimensions. Particular attention is devoted to infinite families of Maxwell-Einstein theories with symmetric and homogeneous target spaces and to Yang-Mills-Einstein theories with compact gauge groups. Extension of the construction to theories with spontaneously-broken gauge symmetry is also discussed.
\end{abstract}

\section{Contents}

1 Introduction and background 1

2 Maxwell-Einstein and Yang-Mills-Einstein supergravities 3

3 Double-copy construction $\quad 7$

4 Amplitudes from the double copy 9

4.1 Generic Jordan family of Maxwell-Einstein supergravities . . . . 9

4.2 Isometry gaugings . . . . . . . . . . . . . . . . . . . . . . . . . . . . .

4.3 Spontaneously-broken theories . . . . . . . . . . . 12

4.4 Homogeneous supergravities . . . . . . . . . . . . 15

5 Discussion and outlook $\quad 18$

\section{Introduction and background}

Over the previous decade, scattering amplitudes in quantum field theories involving gravity have been the object of renewed interest and intense investigation. Calculations in the maximal and half-maximal supergravities have brought into focus simpler-than-expected structures and revealed improved ultraviolet (UV) behaviors. While most of the explicit computations thus far have been within the purview of theories with a large number of supersymmetries, a growing research direction aims to extending this progress to theories with reduced supersymmetry. In this article, I will discuss the extension of modern computational techniques, most prominently the double-copy construction, to infinite families of supergravities with eight supercharges. 
The canonical work of Kawai, Lewellen and Tye (KLT) [1] has first established that tree-level gauge-theory amplitudes are sufficient for constructing tree-level amplitudes in the gravity theories which can be obtained from toroidal compactifications of string theory. The structure underlying the KLT relations has achieved a more modern formulation through the work of Bern, Carrasco and Johansson (BCJ), who expressed loop-level gravity amplitudes at the integrand level as "double copies" of amplitudes in suitably-chosen gauge theories [2, 3]. Their construction relies on the availability of gauge-theory amplitudes in which color and kinematic factors obey a duality known as color/kinematics (C/K) duality. This double-copy procedure constitutes a breath-taking computational advance, as it directly relates loop-level gravity amplitudes with gaugetheory amplitudes, which are significantly easier to obtain. At the same time, the double copy has proven itself to be particularly well-suited for studying amplitudes in broader classes of theories with respect to its KLT precursor.

A combination of the KLT property with unitarity-based methods [4] and the BCJ double-copy construction have both been instrumental in conducting impressive multi-loop computations in the gravitational theories that result most amenable to perturbative calculations, that is theories which possess a large number of supersymmetries. The theory with a maximal number of supersymmetries, $\mathcal{N}=8$ supergravity, plays a key role in this context. This theory was first constructed by Cremmer and Julia and by de Wit and Nicolai 5 7]. Explicit computations have shown that its UV behavior matches the one of the $\mathcal{N}=4$ super-Yang-Mills (sYM) theory at least through four loops [8 11]. These results lend support to the conjecture that the theory might be perturbatively UV-finite in four dimensions [12] and thus constitute the first known example of a mathematically-consistent quantum field theory of gravity 1

Similar calculations have also been carried out for the half-maximal theory $\mathcal{N}=4$ supergravity 13 17] - and more recently for $\mathcal{N}=5$ supergravity [18]. In the absence of additional matter, the former theory appears to be finite at three loops in four dimensions and to diverge at four loops. The latter theory is UVfinite through at least four loops. At the moment, there are no widely-accepted symmetry arguments explaining the finiteness of pure $\mathcal{N}=4$ supergravity at three loops and of $\mathcal{N}=5$ supergravity at four loops. The improved UV behavior of these theories is linked to the presence of enhanced cancellations between different terms of a diagrammatic presentation of the amplitude. Interestingly, the four-loop divergence of pure $\mathcal{N}=4$ supergravity appears to be related to a $U(1)$ quantum anomaly [16, 19]. Additionally, recent calculations in gravity same-helicity amplitudes have shown that adding evanescent operators to the gravity action can alter the UV divergence of the theory while keeping the amplitude's dependence on the renormalization scale unchanged [20]. This result suggests that the latter quantity should be the one regarded as the physically relevant.

\footnotetext{
${ }^{1}$ Reviewing arguments for and against finiteness is beyond the scope of this note. Ultimately, calculations at even higher loop order are necessary to establish conclusively the UV properties of the theory.
} 
A better understanding of enhanced cancellations, the link between anomalies and divergences, and the role of evanescent contributions will be critical in exploring the UV properties of $\mathcal{N}=8$ supergravity and in determining its fate as a potential theory of quantum gravity, at least in the perturbative context. In turn, achieving this understanding requires the ability of conducting perturbative calculations in more general gravity theories, i.e. in theories that are not as special as maximal or half-maximal supergravity. Unsurprisingly, one of the major research directions established since the advent of the double-copy construction has been its extension and application to broader arrays of theories. In particular, it is natural to ask: Is the double-copy structure a general property of gravitational interactions?

Earlier studies on double copies with reduced $(\mathcal{N}<4)$ supersymmetry have investigated very special theories, such as the ones that can be obtained as truncations of $\mathcal{N}=8$ supergravity 21 23], pure supergravities 24], and Einstein gravity coupled to a dilaton and an antisymmetric tensor [25]. This review focuses on extending the construction to infinite classes of supergravities with eight supercharges $-\mathcal{N}=2$ theories in four and five spacetime dimensions. Unlike their more supersymmetric relatives, these theories are no longer completely specified by their matter content alone, in the sense that two theories with the same spectra can have different interaction terms while still preserving $\mathcal{N}=2$ supersymmetry. Moreover, many of these theories cannot be obtained taking field-theory limits of toroidal compactifications of string theory. The aim of this note is to provide a summary and a pedagogical introduction; the reader should consult refs. 26 28] for a complete treatment. Particular attention will be devoted to discussing how some physical features which can arise in supergravities with reduced supersymmetry, such as the introduction of non-abelian gauge interactions and the supergravity Higgs mechanism, are translated in the double-copy language.

\section{Maxwell-Einstein and Yang-Mills-Einstein su- pergravities}

Maxwell-Einstein theories with $\mathcal{N}=2$ supersymmetry in five dimensions have been explicitly known since the early 80s due to the work of Günaydin, Sierra, and Townsend 29 32]. These theories give the coupling of the gravity multiplet to $n$ matter vector multiplets. To fix the notation, we write their bosonic fields as $\left(h_{\mu \nu}, A_{\mu}^{0}\right) \oplus\left(A_{\mu}^{x}, \phi^{x}\right)$, where $x=1,2, \ldots, n$ and $A_{\mu}^{0}$ is the vector in the gravity multiplet. Their bosonic Lagrangian is

$$
e^{-1} \mathcal{L}=-\frac{1}{2} R-\frac{1}{4} \stackrel{\circ}{a}_{I J} F_{\mu \nu}^{I} F^{J \mu \nu}-\frac{1}{2} g_{x y} \partial_{\mu} \phi^{x} \partial^{\mu} \phi^{y}+\frac{e^{-1}}{6 \sqrt{6}} C_{I J K} \varepsilon^{\mu \nu \rho \sigma \lambda} F_{\mu \nu}^{I} F_{\rho \sigma}^{J} A_{\lambda}^{K},
$$

where the $F_{\mu \nu}^{I}(I=0,1, \ldots, n)$ are abelian field strengths. We note that the symmetric tensor $C_{I J K}$ that appears in the $F \wedge F \wedge A$ term needs to be constant to preserve gauge invariance. The matrices $\stackrel{\circ}{a}_{I J}$ and $g_{x y}$ are functions of 
the physical scalars. The authors of ref. [29] employ an Ansatz for supergravity Lagrangian and supersymmetry transformations which depends on generic functions of the scalar fields and use invariance of the Lagrangian under supersymmetry and closure of the supersymmetry algebra to derive a set of algebraic and differential constraints. The most general solution to these constraints is found introducing an auxiliary ambient space with coordinates $\xi^{I}$ and defining a cubic polynomial $\mathcal{V}(\xi)$ in terms of the $C$-tensor,

$$
\mathcal{V}(\xi) \equiv C_{I J K} \xi^{I} \xi^{J} \xi^{K} .
$$

The cubic polynomial is used to introduce the ambient space metric

$$
a_{I J}(\xi) \equiv-\frac{1}{3} \frac{\partial}{\partial \xi^{I}} \frac{\partial}{\partial \xi^{J}} \ln \mathcal{V}(\xi)
$$

Then, the matrices $\stackrel{\circ}{a}_{I J}$ and $g_{x y}$, as well as the other quantities in the Lagrangian, have a geometrical interpretation:

- The $n$-dimensional target space $\mathcal{M}_{5}$ with coordinates $\phi^{x}$ is defined as the hypersurface with

$$
\mathcal{V}(h)=C_{I J K} h^{I} h^{J} h^{K}=1 \quad, \quad h^{I}=\sqrt{\frac{2}{3}} \xi^{I} ;
$$

- The matrix $\stackrel{\circ}{a}_{I J}(\phi)$ which appears in the kinetic-energy term for the vector fields is the restriction of the ambient-space metric to $\mathcal{M}_{5}$,

$$
\stackrel{\circ}{a}_{I J}(\phi)=\left.a_{I J}\right|_{\mathcal{V}(h)=1}
$$

- The metric $g_{x y}(\phi)$ in the kinetic-energy term for the scalars is the induced metric on $\mathcal{M}_{5}$,

$$
g_{x y}(\phi)=\left.\frac{3}{2} \frac{\partial \xi^{I}}{\partial \phi^{x}} \frac{\partial \xi^{J}}{\partial \phi^{y}} a_{I J}\right|_{\mathcal{V}(h)=1} .
$$

The key result is that all quantities in the Maxwell-Einstein Lagrangian can be expressed in terms of the $C$-tensor. Since the $C$-tensor can be obtained by inspecting three-point amplitudes, $\mathcal{N}=2$ Maxwell-Einstein theories in five dimensions are uniquely specified by their three-point interactions. This is in contrast to Maxwell-Einstein theories that only exist in four dimensions, for which supersymmetry is not as constraining.

Requiring positive-definiteness of the scalar and vector kinetic terms at a base-point imposes a constraint relating the base-point $\xi^{I}=c^{I}$ and the $C$ tensor,

$$
c^{I}=\sqrt{\frac{2}{3}} C_{I J K} c^{J} c^{K} .
$$

Choosing the pull-back of the $C$-tensor to be covariantly-constant results in a locally-symmetric target space. In turn, $C$-tensors with this property can be 
obtained using the theory of Jordan algebras and identifying $\mathcal{V}(\xi)$ as the norm of a euclidean Jordan algebra of degree three. This construction has permitted to obtain two classes of supergravities based on symmetric target spaces. The first, named generic Jordan family, is based on an infinite family of reducible Jordan algebras. The second is based on hermitian $3 \times 3$ matrices with entries in the four division algebras $\mathbb{R}, \mathbb{C}, \mathbb{H}$, and $\mathbb{O}$, and gives the so-called Magical Supergravities.

We give here only the cubic polynomials relevant to the cases of interest. Theories belonging to the generic Jordan family have 29]

$$
\mathcal{V}(\xi)=\sqrt{2}\left(\xi^{0}\left(\xi^{1}\right)^{2}-\xi^{0}\left(\xi^{i}\right)^{2}\right), \quad i=2,3, \ldots, n .
$$

Their target spaces in five and four dimensions are the symmetric spaces

$$
\mathcal{M}_{5}=\frac{S O(n-1,1)}{S O(n-1)} \times S O(1,1), \quad \mathcal{M}_{4}=\frac{S O(n, 2)}{S O(n) \times S O(2)} \times \frac{S U(1,1)}{U(1)} .
$$

A more general class of theories is associated to homogeneous target spaces. Van Proeyen and de Wit 33] have shown that the requirement of a transitive group of target-space isometries permits to write the cubic polynomial as

$$
\mathcal{V}(\xi)=\sqrt{2}\left(\xi^{0}\left(\xi^{1}\right)^{2}-\xi^{0}\left(\xi^{i}\right)^{2}\right)+\xi^{1}\left(\xi^{\alpha}\right)^{2}+\tilde{\Gamma}_{\alpha \beta}^{i} \xi^{i} \xi^{\alpha} \xi^{\beta},
$$

where $i, j=2,3, \ldots, q+2, \alpha, \beta$ are indices with range $r$, and the total number of vector multiplets in $5 D$ is $n=2+q+r$. The matrices $\tilde{\Gamma}_{\alpha \beta}^{i}$ are symmetric gamma matrices and form a representation of the euclidean Clifford algebra $\mathcal{C}(q+1,0)$. The parameter $r$ is a multiple of the dimension of the irreducible representation of $\mathcal{C}(q+1,0)$, which is denoted as $\mathcal{D}_{q}$,

$$
r(P, \dot{P}, q)=\mathcal{D}_{q}(P+\dot{P}) \text { if } q=0,4(\bmod 8), \quad r(P, q)=\mathcal{D}_{q} P \text { otherwise, }
$$

where $P, \dot{P}$ are non-negative integers. The parameter $\dot{P}$ is introduced for the values of $q$ such that there exist two inequivalent irreducible representations of $\mathcal{C}(q+1,0)$. The generic Jordan family corresponds to either $q=1$ and $P$ arbitrary or to $P=0$ and $q$ arbitrary. The magical theories correspond to $P=1, \dot{P}=0$, and $q=1,2,4,8$ and, in four dimensions, have the target spaces

$$
\begin{aligned}
\mathcal{M}_{4}\left(J_{3}^{\mathbb{R}}\right)=\frac{S p(6, \mathbb{R})}{U(3)}, & \mathcal{M}_{4}\left(J_{3}^{\mathbb{H}}\right)=\frac{S O^{*}(12)}{U(6)}, \\
\mathcal{M}_{4}\left(J_{3}^{\mathbb{C}}\right)=\frac{S U(3,3)}{S(U(3) \times U(3))}, & \mathcal{M}_{4}\left(J_{3}^{\mathbb{Q}}\right)=\frac{E_{7(-25)}}{E_{6} \times U(1)} .
\end{aligned}
$$

So far we have considered only supergravities of the Maxwell-Einstein type. However, it is possible to promote a subgroup of the isometry group and/or of the R-symmetry group to a non-abelian gauge group as discussed in refs. [30, 31]. Isometry transformations act linearly on the ambient-space coordinates as

$$
\delta_{\alpha} \xi^{I}=\left(M_{r}\right)^{I} \xi^{J} \alpha^{r}, \quad\left[M_{r}, M_{s}\right]=f_{r s}^{t} M_{t},
$$


where the matrices $M_{r}$ leave the $C$-tensor invariant. The gauging procedure is particularly simple when we consider compact isometry gaugings (i.e. we do not gauge part of the R-symmetry group) and we further restrict to the case in which the vector fields furnish the adjoint representation of the gauge group plus additional singlets (i.e. non-trivial representations other than the adjoint are not present). This class of gaugings is obtained by introducing covariant derivatives and field strengths in the Lagrangian in eq. (2.1),

$$
\begin{aligned}
\mathcal{D}_{\mu} \phi^{x} & =\partial_{\mu} \phi^{x}+g_{s} A_{\mu}^{r} K_{r}^{x}, \quad K_{r}^{x}=-\sqrt{\frac{3}{2}} f^{r I J} h_{I} h^{J x} . \\
\mathcal{F}_{\mu \nu}^{I} & =2 \partial_{[\mu} A_{\nu]}^{I}+g_{s} f_{J K}^{I} A_{\mu}^{J} A_{\nu}^{K},
\end{aligned}
$$

where $g_{s}$ is the gauge coupling constant. The antisymmetric tensors $f^{I J K}$ are equal to the structure constants of the gauge group when the three indices $I, J, K$ assume values corresponding to the vectors promoted to gluons and to zero otherwise. Additionally, we need to covariantize the $F \wedge F \wedge A$ term in eq. (2.1) and add a Yukawa-like term to the fermionic part of the Lagrangian. However, supersymmetry does not require a non-trivial scalar potential, and hence the vacua of the theory are still of the Minkowski class.

This procedure yields large classes of $\mathcal{N}=2$ Yang-Mills-Einstein supergravities with compact gauge groups. According to the choice of base-point, the non-abelian gauge symmetry can be unbroken or spontaneously-broken. This review focuses on Yang-Mills-Einstein theories of the generic Jordan family, for which the gauge group is a subgroup of $S O(n)$. All gauge groups can be accommodated in this construction, provided that $n$ is taken to be large enough. The canonical choice of base-point is,

$$
c_{V_{s}}^{I}=\left(\frac{1}{\sqrt{2}}, 1, V_{s}, 0,0\right) .
$$

With $V_{s}=0$, the Yang-Mills-Einstein theory is in the unbroken-gauge phase. When $V_{s} \neq 0$, the theory is on the Coulomb branch and contains massive vector multiplets transforming in matter (non-adjoint) representations of the unbroken gauge group. A $S O(n)$ transformation can be employed to bring all base-points to the form (2.16). Since, in general, the theory will be invariant only under the subgroup of $S O(n)$ which is gauged, this transformation will change the form of the structure constants $f^{I J K}$.

It is convenient to label the massive vectors with an index $\alpha$ running over all (not necessarily irreducible) matter representations, so that the bosonic spectrum of the theory becomes

$$
\left(A_{\mu}^{a}, \phi^{a}\right) \oplus W_{\alpha \mu} \oplus \bar{W}_{\mu}^{\alpha} .
$$

The fields $W_{\alpha \mu}$ and $\bar{W}_{\mu}^{\alpha}$ transform in conjugate representations. This choice corresponds to re-defining the gauge-group generators that do not commute with $T^{2}$ so that $\left(T^{\alpha}\right)^{\dagger}=T_{\alpha}$ in a similar fashion to the treatment of root generators 
in the Chevalley basis of a simple Lie group. The mass matrix for the massive vector multiplets is proportional to the preferred $U(1)$ generator corresponding to the direction singled out by the non-zero $V_{s}$,

$$
m_{\beta}^{\alpha}=i g_{s} \frac{V_{s}}{\sqrt{1-V_{s}^{2}}}\left(f_{\beta}^{2}{ }^{\alpha}\right) .
$$

The following sections will discuss how this plethora of theories can be obtained from the double copy of suitably-chosen gauge theories. It is important to point out that several theories of interest have not (for now) been considered. These include theories with some matter hypermultiplets, supergravities with Rsymmetry gaugings, theories with non-compact gauge groups, five-dimensional supergravities with massive tensors in matter representations, and theories with spontaneously-broken supersymmetry.

\section{Double-copy construction}

We start by considering $L$-loop, $n$-point amplitudes in a gauge theory with fields in the adjoint representation and in a set of matter representations. For very large classes of theories, such amplitudes can be expressed in a form based on a set of cubic graphs in which all external or internal lines are labeled by a gauge-group representation,

$$
\mathcal{A}_{n}^{(L)}=i^{L-1} g^{n-2+2 L} \sum_{i \in \text { cubic }} \int \frac{d^{L D} \ell}{(2 \pi)^{L D}} \frac{1}{S_{i}} \frac{c_{i} n_{i}}{D_{i}} .
$$

$S_{i}$ are symmetry factors relevant for loop amplitudes. The color factors $c_{i}$ are obtained by contracting the invariant tensors with indices in the representations carried by the lines joining at each vertex. The denominators $D_{i}$ are given by the products of propagators associated to the internal lines of the graph. Finally, the numerator factors $n_{i}$ are functions of loop and external momenta, as well as polarization vectors (or spinor-helicity brackets) and, when applicable, invariant tensors of the global-symmetry group.

Color/kinematics duality [2, 3] is the requirement that the color factors in eq. (3.1) have the same algebraic properties as the numerator factors. In particular, due to the gauge-group Jacobi relations and to the representation-matrices commutation relations, there will be triplets of graphs whose color factors add to zero. In this case, an amplitude presentation is said to obey $\mathrm{C} / \mathrm{K}$ duality manifestly if the corresponding numerator factors obey the same relations,

$$
n_{i}-n_{j}=n_{k} \quad \Leftrightarrow \quad c_{i}-c_{j}=c_{k} .
$$

This formulation requires the gauge group to be kept general since avoiding a specific choice for the gauge group prevents the color factors from obeying extra identities aside from the ones stemming from the Jacobi and commutation relations. 
The existence of $\mathrm{C} / \mathrm{K}$-satisfying amplitude presentations has been proven at tree level for $\mathcal{N}=4 \mathrm{sYM}$ [34 36] and, by extension, for some other theories that can be obtained directly from string theory, such as pure sYM theories with various amount of supersymmetry. While at loop level this property has a conjectural status, there is a strong and growing body of evidence in its favor, at least in particular theories. For $\mathcal{N}=4 \mathrm{sYM}$, amplitude presentations which obey the duality manifestly have been constructed up to four loops at four points [10] and up to two loops at five points [37]. General methods for constructing BCJ numerators at one loop have been investigated in refs. [38].

This property has also been established for a variety of other theories, including self-dual Yang-Mills (YM) [40, 41], theories with higher-dimension operators 42], QCD (including supersymmetric versions) [43, 44], non-supersymmetric YM theories with various matter [45], the non-linear sigma model [46], the Bagger-Lambert-Gustavsson theory in three dimensions [47 49], and several non-supersymmetric theories that we will later review.

Duality-satisfying structures arise naturally in string theory [34, 35]. Indeed, the point-particle limit of string theory can be used as a method to construct duality-satisfying numerators $36,50-55]$.

Note that gauge-theory amplitudes will obey the Ward identities dictated by gauge invariance, that is, when a gluon polarization vector for the $i$-th external particle is changed as $\epsilon_{i} \rightarrow \epsilon_{i}+f(k, \epsilon) k_{i}$, the corresponding amplitude will be unchanged. At tree level, this requires the variations of the numerator factors to obey,

$$
n_{i} \rightarrow n_{i}+\Delta_{i}, \quad \sum_{i \in \text { cubic }} \frac{c_{i} \Delta_{i}}{D_{i}}=0 .
$$

This identity relies only on the algebraic relations between the color factors.

We now turn to the double-copy prescription for amplitudes in theories involving gravity and consider pairs or gauge theories with identical gauge groups and the same set of representations. A gravity asymptotic state is associated to each gauge-invariant bilinear build with a pair of states from the two gauge theories. Some states in the gravity theory, most notably the physical polarizations of the graviton and the other states in the same multiplet, will be associated to bilinears with adjoint gauge-theory states. For theories involving non-adjoint matter representations, there will be additional sectors corresponding to tensor products involving conjugate matter representations. Due to the requirement of gauge invariance, bilinears made of one adjoint and one non-adjoint state will be disregarded.

Once the double-copy has been established at the level of the free theory, amplitudes in the interacting theory can be obtained replacing the color factors of the first theory with the numerator factors of the second,

$$
\mathcal{M}_{n}^{(L)}=i^{L-1}\left(\frac{\kappa}{2}\right)^{n-2+2 L} \sum_{i \in \text { cubic }} \int \frac{d^{L D} \ell}{(2 \pi)^{L D}} \frac{1}{S_{i}} \frac{n_{i} \tilde{n}_{i}}{D_{i}} .
$$

Gauge invariance under linearized diffeomorphisms descends from the gauge invariance of the gauge theories entering the construction. The corresponding 
Ward identity dictates that replacing the graviton polarization tensor for the $i$ th external particle as $\epsilon_{\mu \nu}^{i} \rightarrow \epsilon_{\mu \nu}^{i}+f(k, \epsilon) k_{\mu}^{i} q_{\nu}+f(k, \epsilon) k_{\nu}^{i} q_{\mu}$, with $q \cdot k^{i}=0$, does not change the amplitude. However, this follows directly from eq. (3.2) since color factors are replaced with numerator factors that have the same algebraic properties, as required by $\mathrm{C} / \mathrm{K}$ duality. While both gauge theories need to obey $\mathrm{C} / \mathrm{K}$ duality, the construction leads to a sensible theory as long as the numerators of one of the two theories obey the duality manifestly.

When a gauge theory possesses a global symmetry, that symmetry will be inherited by the gravity theory from the double copy. However, the symmetry that is manifest in the construction will be only a subgroup of the symmetry of the resulting gravitational theory. In particular, amplitudes are covariant only under a compact subgroup of the $U$-duality group, while the non-compact symmetries of the Lagrangian manifest themselves through the amplitudes' vanishing soft-limits [56, 57]. An alternative approach to understanding the gauge-theory origin of gravitational symmetries requires a formulation the double copy at the level of off-shell linearized supermultiplets [58 60].

At tree level, the double-copy construction is equivalent to the KLT relations for theories that can be seen as the field-theory limit of some toroidal compactification of string theory. However, the KLT relations do not apply to theories not belonging to this class or, alternatively, when the gauge theories entering the construction have non-adjoint fields.

The double-copy property is a feature of other modern approaches to scattering amplitudes, including most notably the scattering equation formalism 61 63. Beyond the realm of scattering amplitudes, a growing body of literature has identified double-copy structures in the context of classical solutions. The reader should consult refs. 64 68] for exciting developments in this direction. Finally, a comprehensive review of gauge-gravity relations can be found in ref. [69].

\section{Amplitudes from the double copy}

\subsection{Generic Jordan family of Maxwell-Einstein supergrav- ities}

This section applies the double-copy prescription to the specific theories we have introduced in sec. 2 At tree level and for a modest number of external particles, it is still possible, although increasingly cumbersome, to obtain amplitudes with a conventional Feynman-rule computation. For technical reasons, it is easier to conduct computations in four spacetime dimensions. Hence, we start by dimensionally reducing the Lagrangian (2.1). Denoting as $e^{2 \sigma}, A_{\mu}^{-1}$, and $A^{I}$ the $g_{55}$ component of the metric, the graviphoton field from dimensional reduction, and the components of the vector fields along the fifth dimension, the bosonic 
Lagrangian reduced to four dimensions is expressed as follows [70]:

$$
\begin{aligned}
e^{-1} \mathcal{L}^{4 D}=-\frac{1}{2} R-\frac{1}{16} e^{3 \sigma} F_{\mu \nu}^{-1} F^{-1 \mu \nu}-\frac{3}{4} \partial_{\mu} \sigma \partial^{\mu} \sigma-\frac{3}{4} \stackrel{\circ}{a}_{I J} \partial_{\mu} h^{I} \partial^{\mu} h^{J} \\
-\frac{1}{2} e^{-2 \sigma} \stackrel{\circ}{a}_{I J} \partial_{\mu} A^{I} \partial^{\mu} A^{J}-\frac{1}{4} e^{\sigma} \stackrel{o}{a}_{I J}\left(F_{\mu \nu}^{I}-\frac{1}{\sqrt{2}} F_{\mu \nu}^{-1} A^{I}\right)\left(F^{J \mu \nu}-\frac{1}{\sqrt{2}} F^{-1 \mu \nu} A^{J}\right) \\
+\frac{e^{-1}}{2 \sqrt{6}} C_{I J K} \epsilon^{\mu \nu \rho \sigma}\left\{F_{\mu \nu}^{I} F_{\rho \sigma}^{J} A^{K}-\frac{1}{\sqrt{2}} F_{\mu \nu}^{I} F_{\rho \sigma}^{-1} A^{J} A^{K}+\frac{1}{6} F_{\mu \nu}^{-1} F_{\rho \sigma}^{-1} A^{I} A^{J} A^{K}\right\}
\end{aligned}
$$

The real scalars from the five-dimensional Lagrangian pair with the components of the vectors along the fifth dimension to give complex scalars in four dimensions,

$$
z^{I}=\frac{1}{\sqrt{2}} A^{I}+\frac{\sqrt{3}}{2} i e^{\sigma} h^{I} .
$$

An alternative route to write the four-dimensional Lagrangian involves a symplectic formulation based on a prepotential which, in turn, depends on the $C$-tensor, as explained in ref. [71].

Focusing on the case of the generic Jordan family with cubic polynomial (2.4), we expand around the base-point (2.16) and, following ref. [26], take the following steps:

1. Dualize the graviphoton field $A_{\mu}^{-1}$;

2. Redefine the vector fields as

$$
\begin{aligned}
A_{\mu}^{-1} & \rightarrow \frac{1}{4}\left(A_{\mu}^{-1}-A_{\mu}^{0}-\sqrt{2} A_{\mu}^{1}\right), \\
A_{\mu}^{0} & \rightarrow \frac{1}{2}\left(-A_{\mu}^{-1}+A_{\mu}^{0}-\sqrt{2} A_{\mu}^{1}\right) \\
A_{\mu}^{1} & \rightarrow-\frac{1}{\sqrt{2}}\left(A_{\mu}^{-1}+A_{\mu}^{0}\right)
\end{aligned}
$$

3. Dualize the new $A_{\mu}^{1}$ field and redefine $z^{1} \rightarrow-i z^{1}$.

Amplitudes at three and four points can be straightforwardly obtained with a standard Feynman-rule computation. One of the advantages of working in four dimensions is the possibility of employing the spinor-helicity formalism [72]. As an example, the vector-vector-scalar amplitude is

$$
\mathcal{M}_{3}^{(0)}\left(1 A_{-}^{0}, 2 A_{-}^{A}, 3 \bar{z}^{B}\right)=-\frac{\kappa}{2}\langle 12\rangle^{2} \delta^{A B} .
$$

The large symmetry group of the supergravities belonging to the generic Jordan family facilitates the identification of the correct gauge theories entering their double-copy construction. First, one of the gauge theories needs to provide the desired amount of supersymmetry. Hence, a natural candidate is a pure 
$\mathcal{N}=2 \mathrm{sYM}$ theory. As before, we write only the bosonic part of the Lagrangian in four dimensions,

$$
\mathcal{L}_{\text {bos }}^{\mathcal{N}=2}=-\frac{1}{4} F_{\mu \nu}^{\hat{A}} F^{\hat{A} \mu \nu}+\left(\overline{D_{\mu} \phi}\right)^{\hat{A}}\left(D^{\mu} \phi\right)^{\hat{A}}+\frac{g^{2}}{2} f^{\hat{A} \hat{B} \hat{C}} \phi^{\hat{B}} \bar{\phi}^{\hat{C}} f^{\hat{A} \hat{D} \hat{E}} \phi^{\hat{D}} \bar{\phi}^{\hat{E}}
$$

where $\hat{A}, \hat{B}, \hat{C}$ are adjoint gauge indices and $\phi$ is a complex scalar. The second gauge theory is the dimensional reduction of a $(4+n)$-dimensional pure YM theory,

$$
\mathcal{L}^{\mathcal{N}=0}=-\frac{1}{4} F_{\mu \nu}^{\hat{A}} F^{\hat{A} \mu \nu}+\frac{1}{2}\left(D_{\mu} \phi^{A}\right)^{\hat{A}}\left(D^{\mu} \phi^{B}\right)^{\hat{A}}-\frac{g^{2}}{4} f^{\hat{A} \hat{B} \hat{C}} \phi^{\hat{B} B} \phi^{\hat{C} C} f^{\hat{A} \hat{D} \hat{E}} \phi^{\hat{D} B} \phi^{\hat{E} C},
$$

where $\phi^{A}$ are real scalars labeled by the global indices $A, B, C=1, \ldots, n$. This theory has a manifest $S O(n)$ global symmetry. In this case, both gauge theories are known to obey $\mathrm{C} / \mathrm{K}$ duality at least at tree level. However, in the more involved examples discussed in the next subsections, $\mathrm{C} / \mathrm{K}$ duality will pose nontrivial constraints.

At this point, it is straightforward to check that the three-point amplitudes from the double-copy formula (3.3) reproduce the ones from the Lagrangian, provided that we identify the supergravity states with the $(\mathcal{N}=2) \otimes(\mathcal{N}=0)$ bilinears as [26]:

$$
\begin{aligned}
A_{-}^{-1}=\bar{\phi} \otimes A_{-}, & h_{-}=A_{-} \otimes A_{-}, \\
A_{-}^{0}=\phi \otimes A_{-}, & i \bar{z}^{0}=A_{+} \otimes A_{-}, \\
A_{-}^{A}=A_{-} \otimes \phi^{A}, & i \bar{z}^{A}=\bar{\phi} \otimes \phi^{A},
\end{aligned}
$$

with similar expressions for the CPT-conjugate states. With this map, the amplitude (4.4) is obtained as

$$
\mathcal{M}_{3}^{(0)}\left(1 A_{-}^{0}, 2 A_{-}^{A}, 3 \bar{z}^{B}\right)=\left.\left.\mathcal{A}_{3}^{(0)}\left(1 \phi, 2 A_{-}, 3 \bar{\phi}\right)\right|_{\mathcal{N}=2} \otimes \mathcal{A}_{3}^{(0)}\left(1 A_{-}, 2 \phi^{A}, 3 \phi^{B}\right)\right|_{\mathcal{N}=0}
$$

The purpose of the dualization and field redefinitions enacted after reducing the theory to four dimensions has been precisely to "align" the physical states from the supergravity Lagrangian with the ones from the double copy. Since the three-point amplitudes from the double copy match the ones from the Lagrangian, the construction will give the amplitudes of the correct theory also at higher point, at least at tree level (provided that we are able to find amplitude presentations obeying $\mathrm{C} / \mathrm{K}$ duality manifestly). As explained before, this is a consequence of supersymmetry and of the existence of a five-dimensional uplift for the theories.

From a gauge-theory perspective, there are several interaction terms we can envisage adding to the Lagrangians (4.5) and (4.6). Indeed, the following subsections will discuss simple deformations of the gauge theories which have interesting interpretations from the vintage point of the resulting supergravity. 


\subsection{Isometry gaugings}

A very natural deformation of the non-supersymmetric Lagrangian (4.6) is the introduction of cubic scalar couplings of the form,

$$
\delta \mathcal{L}^{\mathcal{N}=0}=\frac{\lambda}{3 !} g f^{\hat{A} \hat{B} \hat{C}} F^{A B C} \phi^{\hat{A} A} \phi^{\hat{B} B} \phi^{\hat{C} C} .
$$

Here $F^{A B C}$ are antisymmetric tensors with three global indices and $\lambda$ is a real parameter. It is important to verify that the theory still obeys $\mathrm{C} / \mathrm{K}$ duality after adding the term (4.9). At four point, the order- $\lambda^{2}$ part of the four-scalar amplitude has expression

$$
\begin{gathered}
\left.\mathcal{A}_{4}^{(0)}\left(1 \phi^{A_{1}}, 2 \phi^{A_{2}}, 3 \phi^{A_{3}}, 4 \phi^{A_{4}}\right)\right|_{\lambda^{2}}=g^{2} \lambda^{2}\left(\frac{1}{s} F^{A_{1} A_{2} B} F^{A_{3} A_{4} B} f^{\hat{A}_{1} \hat{A}_{2} \hat{B}} f^{\hat{A}_{3} \hat{A}_{4} \hat{B}}+\right. \\
\left.\frac{1}{u} F^{A_{3} A_{1} B} F^{A_{2} A_{4} B} f^{\hat{A}_{3} \hat{A}_{1} \hat{B}} f^{\hat{A}_{2} \hat{A}_{4} \hat{B}}+\frac{1}{t} F^{A_{2} A_{3} B} F^{A_{1} A_{4} B} f^{\hat{A}_{2} \hat{A}_{3} \hat{B}} f^{\hat{A}_{1} \hat{A}_{4} \hat{B}}\right) .
\end{gathered}
$$

Requiring that this amplitude obeys the duality produces a non-trivial constraint on the $F$-tensors, which need to obey Jacobi relations. We have verified up to six points that $\mathrm{C} / \mathrm{K}$ duality does not pose additional constraints in the YM-scalar theory deformed by the term (4.9) 26].

The interaction term (4.9) produces a non-vanishing amplitude between three scalars. Taking the double copy of this amplitude with a three-gluon amplitude in the supersymmetric gauge theory produces a non-vanishing gravity amplitude between three vector fields,

$$
\mathcal{M}_{3}^{(0)}\left(1 A_{-}^{A}, 2 A_{-}^{B}, 3 A_{+}^{C}\right)=-\left(\frac{\kappa}{2}\right) \frac{\lambda}{\sqrt{2}} \frac{\langle 12\rangle^{3}}{\langle 23\rangle\langle 31\rangle} F^{A B C} .
$$

Hence, the interaction term (4.9) produces non-abelian gauge interactions in the supergravity Lagrangian [26]. Inspection of the amplitudes from the double-copy leads to the identification of $F^{A B C}$ and $\lambda$ with the supergravity gauge-group structure constants and coupling constant,

$$
\lambda\left(\frac{\kappa}{2}\right) F^{A B C}=2 g_{s} f_{\mathrm{sg}}^{A B C}, \quad A, B, C=1, \ldots, n .
$$

It is interesting to note that, in this case, the double copy has promoted a global symmetry in one of the gauge theories to a local symmetry in the resulting supergravity.

This construction extends previous single-trace amplitude results from ref. 73. Amplitudes in Yang-Mills-Einstein theories have also been recently investigated from the point of view of scattering equations 74,75 ], ambitwistor string constructions [76, 77], and string amplitudes [78 81].

\subsection{Spontaneously-broken theories}

Having formulated a double-copy construction for Yang-Mills-Einstein theories, it is paramount to probe its validity away from the unbroken-gauge phase, i.e. 
for $V_{s} \neq 0$. In this case, a sensible candidate for the first gauge theory is a spontaneously-broken version of the Lagrangian (4.5). This is achieved by considering a scalar vacuum expectation value of the form

$$
\left\langle\phi^{a}\right\rangle=V t^{0} \delta^{a 0}
$$

with $V$ real. Reality of the expectation value is a consequence of the existence of a five-dimensional uplift for the spontaneously-broken theory. We then write the gauge-group generators as $\left\{t^{\hat{A}}\right\}=\left\{t^{\hat{a}}, t^{\hat{\alpha}}, t_{\hat{\alpha}}\right\}$, where $\left(t^{\hat{a}}\right)^{\dagger}=t^{\hat{a}}$, $\left(t_{\hat{\alpha}}\right)^{\dagger}=t^{\hat{\alpha}}$, and the index $\hat{a}$ runs over all unbroken generators. Scalar and vector fields of the resulting spontaneously-broken gauge theory split accordingly as $A_{\mu}^{\hat{A}}=\left(A_{\mu}^{\hat{a}}, W_{\mu \hat{\alpha}}, \bar{W}_{\mu}^{\hat{\alpha}}\right)$ and $\phi^{\hat{A}}=\left(\phi^{\hat{a}}, \varphi_{\hat{\alpha}}, \bar{\varphi}^{\hat{\alpha}}\right)$. With this notation, the structure constants $f^{\hat{A} \hat{B} \hat{C}}$ of the gauge group before symmetry breaking yield the structure constants of the unbroken gauge group, representation matrices for the massive vector multiplets, and Clebsh-Gordan coefficients entering couplings between three matter representations,

$f^{\hat{a} \hat{b} \hat{c}}=-i \operatorname{Tr}\left(\left[t^{\hat{a}}, t^{\hat{b}}\right] t^{\hat{c}}\right), \quad f_{\hat{\beta}}^{\hat{a} \hat{\alpha}}=-i \operatorname{Tr}\left(\left[t^{\hat{a}},\left(t^{\hat{\beta}}\right)^{\dagger}\right] t^{\hat{\alpha}}\right), \quad f_{\hat{\beta}}^{\hat{\alpha} \hat{\gamma}}=-i \operatorname{Tr}\left(\left[t^{\hat{\alpha}},\left(t^{\hat{\beta}}\right)^{\dagger}\right] t^{\hat{\gamma}}\right)$.

The tensors (4.14) obey color relations inherited from the Jacobi identities of the gauge group before symmetry breaking. Aside from the Jacobi relations for the structure constants of the unbroken gauge group and the commutation relations for the representation matrices, the Clebsh-Gordan coefficients obey two extra relations,

$$
\begin{aligned}
& f_{\hat{\epsilon}}^{\hat{\alpha} \hat{\gamma}} f_{\hat{\delta}}^{\hat{\epsilon} \hat{\beta}}-f_{\hat{\epsilon}}^{\hat{\alpha} \hat{\beta}} f_{\hat{\delta}}^{\hat{\epsilon} \hat{\gamma}}=f_{\hat{\delta}}^{\hat{\alpha} \hat{\epsilon}} f_{\hat{\epsilon}}^{\hat{\gamma} \hat{\beta}}, \\
& \left(f_{\hat{\gamma}}^{\hat{\beta} \hat{\epsilon}} f_{\hat{\epsilon} \hat{\delta}}^{\hat{\alpha}}+f_{\hat{\delta}}^{\hat{\alpha} \hat{\epsilon}} f_{\hat{\epsilon} \hat{\gamma}}^{\hat{\beta}}+f_{\hat{\gamma}}^{\hat{a} \hat{\beta}} f_{\hat{\delta}}^{\hat{a} \hat{\alpha}}\right)-(\hat{\alpha} \leftrightarrow \hat{\beta})=f_{\hat{\epsilon}}^{\hat{\alpha} \hat{\beta}} f_{\hat{\delta} \hat{\gamma}}^{\hat{\epsilon}} .
\end{aligned}
$$

These identities arise only for representations obtained from the symmetry breaking of a larger gauge group. The seven-term identity can be thought of as a set of three-term identities since, for any assignment of external masses, at most three terms can be non-zero.

The mass spectrum of the theory is given by $m_{\hat{\alpha}}^{\hat{\beta}}=i g V f_{\hat{\alpha}}^{0 \hat{\beta}}$, which can be taken in a block-diagonal form with blocks corresponding to different irreducible representations. Massive fields can be further organized into representations labeled by the $U(1)$ charge associated to their mass. The number of such representations needs to be kept general in order to cover all possible symmetry-breaking patterns. Amplitudes in the simple supersymmetric theory discussed here can be obtained considering higher-dimensional amplitudes with massless fields and assigning compact momenta proportional to the masses to the external particles, as done e.g. in refs. [82 86].

The non-supersymmetric gauge theory entering the construction is an extension of the YM-scalar theory (4.6). In particular, the theory has a set of complex scalars $\varphi_{\alpha}$ which have the same masses as the ones in the spontaneously-broken 
theory and transform in conjugate representations. We then write the most general cubic couplings involving three scalars, obtaining the Lagrangian:

$$
\begin{aligned}
\mathcal{L}^{\prime}= & -\frac{1}{4} F_{\mu \nu}^{\hat{a}} F^{\mu \nu \hat{a}}+\frac{1}{2}\left(D_{\mu} \phi^{a}\right)^{\hat{a}}\left(D^{\mu} \phi^{a}\right)^{\hat{a}}+\left(\overline{D_{\mu} \varphi^{\alpha}}\right)_{\hat{\alpha}}\left(D^{\mu} \varphi_{\alpha}\right)^{\hat{\alpha}}-\left(m^{2}\right)_{\alpha}^{\beta} \bar{\varphi}_{\hat{\alpha}}^{\alpha} \varphi_{\beta}^{\hat{\alpha}} \\
& +\frac{1}{3 !} g \lambda F^{a b c} f^{\hat{a} \hat{b} \hat{c}} \phi^{a \hat{a}} \phi^{b \hat{b}} \phi^{c \hat{c}}+g \lambda \Delta^{a b} F_{\alpha}^{a}{ }_{\alpha}^{\beta} f_{\hat{\gamma}}^{\hat{a}} \phi^{b \hat{a}} \bar{\varphi}_{\hat{\beta}}^{\alpha} \varphi_{\beta}^{\hat{\gamma}} \\
& +\frac{1}{2} g \lambda F_{\beta}^{\alpha}{ }^{\gamma} f_{\hat{\alpha} \hat{\gamma}}^{\hat{\beta}} \varphi_{\alpha}^{\hat{\alpha}} \bar{\varphi}_{\hat{\beta}}^{\beta} \varphi_{\gamma}^{\hat{\gamma}}+\frac{1}{2} g \lambda F_{\alpha \gamma}^{\beta} f_{\hat{\beta}}^{\hat{\alpha} \hat{\gamma}} \bar{\varphi}_{\hat{\alpha}}^{\alpha} \varphi_{\beta}^{\hat{\beta}} \bar{\varphi}_{\hat{\gamma}}^{\gamma}+\mathcal{L}_{\text {contact }}, \quad(4.16)
\end{aligned}
$$

where $\Delta^{a b}$ is a diagonal matrix and $F^{a b c}, F_{\alpha}^{a \beta}, F_{\beta}^{\alpha}{ }^{\gamma}$ are tensors in the global indices. Imposing the relations (4.15) on the numerators of amplitudes with four massive scalars fixes the contact terms in the Lagrangian (4.16) and produces the following constraints:

- The $F$-tensors need to collectively obey the same algebraic relations as the structure constants, representation matrices, and Clebsh-Gordan symbols of the gauge group. They can be interpreted as pieces of the structure constants of a larger group, which is spontaneously broken down to a subgroup with structure constants $F^{a b c}$. The symmetry-breaking pattern of the global group needs to be the same as the one of the gauge group;

- The massive scalars need to assume a block-diagonal form such that

$$
2 V f_{\hat{\beta}}^{0 \hat{\alpha}} \varphi_{\alpha}^{\hat{\beta}}=\lambda \rho F_{\alpha}^{0 \beta} \varphi_{\beta}^{\hat{\alpha}},
$$

where $\rho$ is a free parameter, i.e. for each block, the charge of the preferred $U(1)$ gauge generator is proportional to the charge of a preferred global $U(1)$ generator;

- The diagonal matrices $\Delta$ are fixed to $\Delta^{a b}=\delta^{a b}+\left(\sqrt{1+\rho^{2}}-1\right) \delta^{a 0} \delta^{0 b}$.

The reader should consult ref. 27] for a complete treatment. As before, identifying supergravity three-point amplitudes from the double-copy (3.3) with the ones from a Feynman-rule computation leads to the $(\mathcal{N}=2) \times(\mathcal{N}=0)$ field map:

$$
\begin{aligned}
A_{-}^{-1}=\bar{\phi} \otimes A_{-}, & h_{-}=A_{-} \otimes A_{-}, \\
A_{-}^{0}=\phi \otimes A_{-}, & i \bar{z}^{0}=A_{+} \otimes A_{-}, \\
A_{-}^{a}=A_{-} \otimes \phi^{a}, & i \bar{z}^{a}=\bar{\phi} \otimes \phi^{a}, \\
\varphi_{\alpha}=\varphi \otimes \varphi_{\alpha}, & W_{\alpha}=W \otimes \varphi_{\alpha} .
\end{aligned}
$$

Similarly, the two free parameters $\lambda$ and $\rho$ in the non-supersymmetric gauge theory are related to the supergravity parameters as

$$
\left(\frac{\kappa}{2}\right) \lambda F^{A B C}=2 g_{s} f_{\mathrm{sg}}^{A B C}, \quad \rho=\frac{V_{s}}{\sqrt{1-V_{s}^{2}}} .
$$




\begin{tabular}{l|c|c} 
Gravity coupled to XM & Left gauge theory & Right gauge theory \\
\hline $\mathcal{N}=4$ YMESG & $\mathcal{N}=4$ SYM & YM $+\phi^{\gamma}$ \\
$\mathcal{N}=2$ YMESG (gen.Jordan) & $\mathcal{N}=2$ SYM & YM $+\phi^{\gamma}$ \\
$\mathcal{N}=0$ YM $_{\mathrm{DR}}-\mathrm{E}+$ dilaton $+B^{\mu \nu}$ & $\mathcal{Y M}_{\mathrm{DR}}$ & $\mathrm{YM}+\phi^{\gamma}$
\end{tabular}

Table 1: Double-copy constructions for spontaneously-broken Yang-Mills-Einstein supergravities with different amounts of supersymmetry [27]. YY $_{\mathrm{DR}}$ indicates a spontaneously-broken YM-scalar theory from dimensional reduction.

Note that the massless supergravity states are obtained as double copies of adjoint gauge-theory states, while the massive sector is obtained from the double copy of gauge-theory states in matter representations. While this review considers supergravities with eight supercharges, analogous constructions can be set forth for spontaneously-broken theories with $\mathcal{N}=4$ or no supersymmetry by adjusting the spontaneously-broken gauge-theory factor (see Table 1)

\subsection{Homogeneous supergravities}

The analysis of the supergravity Higgs mechanism has underlined the key role played by matter (non-adjoint) representations in extending the double copy to larger classes of supergravities. Along these lines, another option for modifying the construction of sec. 4.1 is to include in the non-supersymmetric gauge theory fermions transforming in a matter representation. Adding adjoint fermions without at the same time introducing extra supersymmetries is forbidden by $\mathrm{C} / \mathrm{K}$ duality, as discussed in ref. 22]. To have a non-trivial result, we also need to add to the supersymmetric theory some fields transforming in the conjugate representation. In this regard, the minimal set of fields that can be added is two fermions and two scalars, corresponding to a half-hypermultiplet. To fix notation, we denote the physical states in the supersymmetric gauge theory as

$$
\left(A_{+}^{\hat{a}}, \psi_{+}^{\hat{a}}, \phi^{\hat{a}}\right)_{G} \oplus\left(A_{-}^{\hat{a}}, \psi_{-}^{\hat{a}}, \bar{\phi}^{\hat{a}}\right)_{G} \oplus\left(\chi_{+}, \varphi_{1}, \varphi_{2}, \chi_{-}\right)_{R},
$$

where $R$ and $G$ label the matter representation and the adjoint representation. For generic $R$, we also need to add the CPT-conjugate states. However, here it is convenient to consider the case in which the representation $R$ is pseudo-real, i.e. there exist a unitary matrix $V$ such that $V T^{\hat{a}} V^{\dagger}=-\left(T^{\hat{a}}\right)^{*}, V V^{*}=-1$. In this case, the half-hypermultiplet alone is CPT-self-conjugate.

The non-supersymmetric gauge theory entering the construction is a YMscalar theory with extra fermions in the representation $R$,

$$
\begin{aligned}
\mathcal{L}= & -\frac{1}{4} F_{\mu \nu}^{\hat{a}} F^{\hat{a} \mu \nu}+\frac{1}{2}\left(D_{\mu} \phi^{a}\right)^{\hat{a}}\left(D^{\mu} \phi^{a}\right)^{\hat{a}}+\frac{i}{2} \bar{\lambda}^{\alpha} D_{\mu} \gamma^{\mu} \lambda_{\alpha} \\
& +\frac{g}{2} \phi^{a \hat{a}} \Gamma_{\alpha}^{a} \bar{\lambda}^{\alpha} \gamma_{5} T^{\hat{a}} \lambda_{\beta}-\frac{g^{2}}{4} f^{\hat{a} \hat{b} \hat{e}} f^{\hat{c} \hat{d} \hat{e}} \phi^{a \hat{a}} \phi^{b \hat{b}} \phi^{a \hat{c}} \phi^{b \hat{d}} .
\end{aligned}
$$

$\hat{a}, \hat{b}$ are adjoint indices of the gauge group and $\alpha, \beta=1, \ldots, r$ and $a, b=$ $1, \ldots, q+2$ are global indices. Spacetime spinor indices and gauge-group indices 


\begin{tabular}{ccccc}
$q$ & $\mathcal{D}_{q}$ & $4 D$ fermions $r(q, P, \dot{P})$ & conditions & flavor group \\
\hline-1 & 1 & $P$ & $\mathrm{R}$ & $S O(P)$ \\
0 & 1 & $P+\dot{P}$ & $\mathrm{RW}$ & $S O(P) \times S O(\dot{P})$ \\
1 & 2 & $2 P$ & $\mathrm{R}$ & $S O(P)$ \\
2 & 4 & $4 P$ & $\mathrm{R}$ or $\mathrm{W}$ & $U(P)$ \\
3 & 8 & $8 P$ & PR & $U S p(2 P)$ \\
4 & 8 & $8 P+8 \dot{P}$ & PRW & $U S p(2 P) \times U S p(2 \dot{P})$ \\
5 & 16 & $16 P$ & PR & $U S p(2 P)$ \\
6 & 16 & $16 P$ & R or W & $U(P)$ \\
$k+8$ & $16 \mathcal{D}_{k}$ & $16 r(k, P, \dot{P})$ & as for $k$ & as for $k$
\end{tabular}

Table 2: Parameters in the double-copy construction for homogeneous supergravities [28]. The third column gives the number of $4 D$ irreducible spinors in the nonsupersymmetric gauge theory, which can obey a reality $(\mathrm{R})$, pseudo-reality (PR) or Weyl (W) conditions.

for the representation $R$ are not explicitly displayed. As before, we introduce unconstrained matrices $\Gamma_{\alpha}^{a} \beta$ in the global indices and let $\mathrm{C} / \mathrm{K}$ duality establish their algebraic properties.

Imposing $\mathrm{C} / \mathrm{K}$ duality on the four-point amplitudes with two adjoint scalars and two matter fermions gives the constraint [28]:

$$
n_{u}-n_{t}=n_{s} \quad \rightarrow \quad\left\{\Gamma^{a}, \Gamma^{b}\right\}=2 \delta^{a b},
$$

i.e. that the matrices $\Gamma^{a}$ form a $(q+2)$-dimensional Clifford algebra. In turn, thanks to this relation, the non-supersymmetric theory can be regarded as the dimensional reduction of a YM + fermions theory in $D=(q+6)$ dimensions. The problem of charting all possible supergravities obtained with this construction is thus equivalent to listing irreducible spinor representations in $D=(q+6)$ dimensions. A parameter $P$ is equal to the number of irreducible spinors introduced in the gauge theory. When $P$ is greater than one, an additional flavor symmetry will be manifest in the non-supersymmetric gauge theory. The only difference with the standard treatment of spinors in $D$ dimensions is the presence of the matrix $V$ acting on the gauge indices, which enters reality (R) and pseudo-reality $(\mathrm{PR})$ conditions of the form [28]

$$
\bar{\lambda}=\lambda^{t} \mathcal{C}_{4} C V, \quad \mathrm{R}: C=\mathcal{C}_{q}, \quad \mathrm{PR}: C=\mathcal{C}_{q} \Omega,
$$

where $\mathcal{C}_{q}$ and $\mathcal{C}_{4}$ are the global and spacetime charge-conjugation matrices which obey the relations $\mathcal{C}_{q} \Gamma^{a} \mathcal{C}_{q}^{-1}=-\zeta\left(\Gamma^{a}\right)^{t}, \mathcal{C}_{4} \gamma^{\mu} \mathcal{C}_{4}^{-1}=-\zeta\left(\gamma^{\mu}\right)^{t}, \zeta= \pm 1$. $\Omega$ is an antisymmetric real matrix acting on the flavor indices. These conditions can be employed to obtain irreducible spinor representations with a $q$-by- $q$ analysis. Note that the reality $(\mathrm{R})$ condition is the combination of a pseudo-reality condition on the gauge-group indices with a pseudo-Majorana condition on the spinor indices. In the particular case of $q=0,4(\bmod 8)$, there are two inequivalent irreducible spinors, and the parameters $P, \dot{P}$ count the number of each. 


\begin{tabular}{l|c}
\multicolumn{1}{c|}{ Gauge theories } & Supergravity \\
\hline GT1: Pure $\mathcal{N}=2$ sYM theory & $\begin{array}{c}\text { Generic Jordan family of } \\
\text { Maxwell-Einstein supergravities }\end{array}$ \\
GT2: YM+scalar theory from dim. red. & $\begin{array}{c}\text { Yang-Mills-Einstein theories } \\
\text { (compact gaugings) }\end{array}$ \\
\hline GT1: As before & Higgsed supergravities \\
GT2: Add trilinear scalar couplings & \\
\hline GT1: Spont. broken $\mathcal{N}=2$ sYM theory & Homogeneous supergravities \\
GT2: Add massive scalars & \\
\hline GT1: Add hypers in representation $R$ & \\
GT2: Add fermions in representation $R$ & with Yukawa couplings \\
\hline GT1: Add hypers in representation $R$ & Supergravities with hypermultiplets \\
GT2: Add scalars in representation $R$ &
\end{tabular}

Table 3: Summary of double-copy constructions for supergravities with eight supercharges. The first row describes the basic construction, while rows 2-5 list some variants.

The analysis is summarized in Table 2 The total range of the global indices $\alpha, \beta$ is fixed to $r=\mathcal{D}_{q} P$ or $r=\mathcal{D}_{q}(P+\dot{P})$, where $4 \mathcal{D}_{q}$ is the dimension of the irreducible $S O(q+5,1)$ spinor. The number of vector multiplets in the fourdimensional supergravity theory obtained with the double-copy construction is equal to $(3+q+r)$. This construction reproduces the classification of homogeneous supergravities by de Wit and van Proeyen [33]. As for the previous cases, three-point amplitudes from the double copy (3.3) are compared with the ones from the supergravity Lagrangian. The two sets of amplitudes agree provided that the field map

$$
\begin{aligned}
A_{-}^{-1}=\bar{\phi} \otimes A_{-}, & h_{-}=A_{-} \otimes A_{-}, \\
A_{-}^{0}=\phi \otimes A_{-}, & i \bar{z}^{0}=A_{+} \otimes A_{-}, \\
A_{-}^{a}=A_{-} \otimes \phi^{a}, & i \bar{z}^{a}=\bar{\phi} \otimes \phi^{a}, \\
A_{\alpha-}=\chi_{-} \otimes\left(U \lambda_{-}\right)_{\alpha}, & i \bar{z}_{\alpha}=\chi_{+} \otimes\left(U \lambda_{-}\right)_{\alpha},
\end{aligned}
$$

is employed. $U$ is a unitary matrix whose exact form depends on the choice of $\Gamma^{1}$. With an appropriate choice for $U$, some of the entries of the $\Gamma^{a}$ matrices in the Yukawa couplings of the non-supersymmetric gauge theory reproduce the real $\tilde{\Gamma}^{i}$ matrices in the cubic polynomial (2.10),

$$
\left(U^{t} \Gamma^{a} C^{-1} U\right)=\left(-\mathbf{1}, i \tilde{\Gamma}^{i}\right)
$$

It should be noted that the four magical supergravities can be recovered as particular cases of this construction. Finally, this framework can be modified to include supergravities with hypermultiplets by introducing in the nonsupersymmetric gauge theory scalars transforming in the representation $R$. 


\section{Discussion and outlook}

This note has discussed how the double-copy construction can be formulated in theories with $\mathcal{N}=2$ supersymmetry in four and five dimensions. Our starting point has been an infinite family of supergravities with symmetric target spaces, the generic Jordan family of Maxwell-Einstein theories. In this case, two very simple gauge theories enter the construction: one is a pure sYM theory, the other a YM-scalar theory obtained by dimensional reduction. Simple modifications of the above theories produce intriguing effects in the resulting supergravity. In particular, some physical features of supergravities with reduced supersymmetry, such as the possibility of gauging part of their isometry groups, are straightforwardly incorporated in the double-copy framework. Results are summarized in Table 3 ,

Extension of the double copy has relied on the possibility of incorporating non-adjoint representations in the gauge theories entering the construction. In all cases, it has been possible to identify the supergravity given by the doublecopy construction from its amplitudes at three points. This is a consequence of supersymmetry combined with the existence of a five-dimensional uplift for both the supergravity theory and the gauge theories entering the construction. Nevertheless, amplitudes at higher points have been considered as a consistency check in refs. [26 28], where sample amplitudes at one loop have also been displayed.

These developments open the door to loop-level computations in large families of supergravities with eight supercharges. As UV divergences are expected already at one loop for generic theories with matter, results will likely cast some insight into the abundance and role of enhanced cancellations and into the connection between higher-loop divergences and lower-loop quantum anomalies. A systematic study of loop-level amplitudes is currently ongoing.

The success in extending the double copy strongly suggests that the construction has a significant role to play in computations for generic gravity theories with reduced (or no) supersymmetry. In this respect, the extension of the construction to the supergravity Higgs mechanism is particularly relevant, as spontaneously-broken gauge symmetry is a general feature of Yang-MillsEinstein theories, which generically have non-compact gauge groups that are broken down to a compact subgroup. Finally, the last extension discussed in this article is critically important as homogeneous supergravities now constitute the largest family of theories previously studied in the supergravity literature for which a double-copy construction is explicitly known. Such family includes theories that cannot be obtained as a toroidal compactification of string theory, marking a substantial departure from the setting in which the KLT relations and the double-copy construction were first introduced. 


\section{Acknowledgements}

I am very grateful to Murat Günaydin, Henrik Johansson, and Radu Roiban for collaboration on refs. [26 28], on which this review is based. My research is supported by the German Research Foundation (DFG) through the Collaborative Research Centre "Space-time-matter" (SFB 647, teilgruppe C6).

\section{References}

[1] H. Kawai, D. C. Lewellen and S.-H. H. Tye, "A Relation Between Tree Amplitudes Of Closed And Open Strings, Nucl. Phys. B 269, 1 (1986);

[2] Z. Bern, J. J. M. Carrasco and H. Johansson, "New Relations for Gauge-Theory Amplitudes," Phys. Rev. D 78, 085011 (2008) [arXiv:0805.3993 [hep-ph]].

[3] Z. Bern, J. J. M. Carrasco and H. Johansson, "Perturbative Quantum Gravity as a Double Copy of Gauge Theory," Phys. Rev. Lett. 105, 061602 (2010) arXiv:1004.0476 [hep-th]].

[4] Z. Bern, L. J. Dixon, D. C. Dunbar and D. A. Kosower, "One loop n point gauge theory amplitudes, unitarity and collinear limits," Nucl. Phys. B 425, 217 (1994) hep-ph/9403226.

[5] E. Cremmer and B. Julia, "The $\mathrm{N}=8$ Supergravity Theory. 1. The Lagrangian," Phys. Lett. B 80, 48 (1978).

[6] E. Cremmer and B. Julia, "The SO(8) Supergravity," Nucl. Phys. B 159, 141 (1979).

[7] B. de Wit and H. Nicolai, "N=8 Supergravity," Nucl. Phys. B 208, 323 (1982).

[8] Z. Bern, J. J. Carrasco, L. J. Dixon, H. Johansson, D. A. Kosower and R. Roiban, "Three-Loop Superfiniteness of N=8 Supergravity," Phys. Rev. Lett. 98, 161303 (2007) hep-th/0702112.

[9] Z. Bern, J. J. M. Carrasco, L. J. Dixon, H. Johansson and R. Roiban, "Manifest Ultraviolet Behavior for the Three-Loop Four-Point Amplitude of N=8 Supergravity," Phys. Rev. D 78, 105019 (2008) arXiv:0808.4112 [hep-th]].

[10] Z. Bern, J. J. Carrasco, L. J. Dixon, H. Johansson and R. Roiban, "The Ultraviolet Behavior of N=8 Supergravity at Four Loops," Phys. Rev. Lett. 103, 081301 (2009) arXiv:0905.2326 [hep-th]].

[11] Z. Bern, J. J. M. Carrasco, L. J. Dixon, H. Johansson and R. Roiban, "Simplifying Multiloop Integrands and Ultraviolet Divergences of Gauge Theory and Gravity Amplitudes," Phys. Rev. D 85, 105014 (2012) arXiv:1201.5366] [hepth]].

[12] Z. Bern, L. J. Dixon and R. Roiban, "Is $\mathrm{N}=8$ supergravity ultraviolet finite?," Phys. Lett. B 644, 265 (2007) hep-th/0611086. 
[13] Z. Bern, S. Davies, T. Dennen and Y. -t. Huang, "Absence of Three-Loop FourPoint Divergences in N=4 Supergravity," Phys. Rev. Lett. 108, 201301 (2012) arXiv:1202.3423 [hep-th]].

[14] Z. Bern, S. Davies, T. Dennen and Y. -t. Huang, "Ultraviolet Cancellations in Half-Maximal Supergravity as a Consequence of the Double-Copy Structure," Phys. Rev. D 86, 105014 (2012) arXiv:1209.2472 [hep-th]].

[15] Z. Bern, S. Davies and T. Dennen, "The Ultraviolet Structure of Half-Maximal Supergravity with Matter Multiplets at Two and Three Loops," Phys. Rev. D 88, 065007 (2013) arXiv:1305.4876 [hep-th]].

[16] Z. Bern, S. Davies, T. Dennen, A. V. Smirnov and V. A. Smirnov, "Ultraviolet Properties of N=4 Supergravity at Four Loops," Phys. Rev. Lett. 111, no. 23, 231302 (2013) arXiv:1309.2498 [hep-th]].

[17] Z. Bern, S. Davies and T. Dennen, "The Ultraviolet Critical Dimension of HalfMaximal Supergravity at Three Loops," arXiv:1412.2441 [hep-th].

[18] Z. Bern, S. Davies and T. Dennen, "Enhanced ultraviolet cancellations in $\mathcal{N}=5$ supergravity at four loops," Phys. Rev. D 90, no. 10, 105011 (2014) arXiv:1409.3089 [hep-th]].

[19] J. J. M. Carrasco, R. Kallosh, R. Roiban and A. A. Tseytlin, "On the U(1) duality anomaly and the S-matrix of N=4 supergravity," JHEP 1307, 029 (2013) arXiv:1303.6219 [hep-th]].

[20] Z. Bern, C. Cheung, H. H. Chi, S. Davies, L. Dixon and J. Nohle, "Evanescent Effects Can Alter Ultraviolet Divergences in Quantum Gravity without Physical Consequences," arXiv:1507.06118 [hep-th].

[21] J. J. M. Carrasco, M. Chiodaroli, M. Günaydin and R. Roiban, "One-loop fourpoint amplitudes in pure and matter-coupled $\mathrm{N} \leq 4$ supergravity," JHEP 1303, 056 (2013) arXiv:1212.1146 [hep-th]];

[22] M. Chiodaroli, Q. Jin and R. Roiban, "C/K duality for general abelian orbifolds of N=4 super Yang-Mills theory," JHEP 1401, 152 (2014) arXiv:1311.3600 [hep-th]].

[23] P. H. Damgaard, R. Huang, T. Sondergaard and Y. Zhang, "The Complete KLT-Map Between Gravity and Gauge Theories," JHEP 1208, 101 (2012) arXiv:1206.1577 [hep-th]].

[24] H. Johansson and A. Ochirov, "Pure Gravities via Color-Kinematics Duality for Fundamental Matter," arXiv:1407.4772 [hep-th].

[25] Z. Bern, S. Davies, T. Dennen, Y. -t. Huang and J. Nohle, "ColorKinematics Duality for Pure Yang-Mills and Gravity at One and Two Loops," arXiv:1303.6605 [hep-th].

[26] M. Chiodaroli, M. Günaydin, H. Johansson and R. Roiban, "Scattering amplitudes in $\mathcal{N}=2$ Maxwell-Einstein and Yang-Mills-Einstein supergravity," JHEP 1501, 081 (2015) arXiv:1408.0764 [hep-th]]. 
[27] M. Chiodaroli, M. Gunaydin, H. Johansson and R. Roiban, "Spontaneously Broken Yang-Mills-Einstein Supergravities as Double Copies," arXiv:1511.01740 [hep-th].

[28] M. Chiodaroli, M. Gunaydin, H. Johansson and R. Roiban, "Complete construction of magical, symmetric and homogeneous $\mathrm{N}=2$ supergravities as double copies of gauge theories," Phys. Rev. Lett. 117, no. 1, 011603 (2016) doi:10.1103/PhysRevLett.117.011603 arXiv:1512.09130 [hep-th]].

[29] M. Günaydin, G. Sierra and P. K. Townsend, "The Geometry of N=2 MaxwellEinstein Supergravity and Jordan Algebras," Nucl. Phys. B 242, 244 (1984).

[30] M. Günaydin, G. Sierra and P. K. Townsend, "Gauging the d = 5 MaxwellEinstein Supergravity Theories: More on Jordan Algebras," Nucl. Phys. B 253, 573 (1985).

[31] M. Günaydin, G. Sierra and P. K. Townsend, "Quantization of the Gauge Coupling Constant in a Five-dimensional Yang-Mills / Einstein Supergravity Theory," Phys. Rev. Lett. 53, 322 (1984).

[32] M. Gunaydin, G. Sierra and P. K. Townsend, "More on $d=5$ Maxwell-einstein Supergravity: Symmetric Spaces and Kinks," Class. Quant. Grav. 3, 763 (1986).

[33] B. de Wit and A. Van Proeyen, "Special geometry, cubic polynomials and homogeneous quaternionic spaces," Commun. Math. Phys. 149, 307 (1992) hep-th/9112027.

[34] N. E. J. Bjerrum-Bohr, P. H. Damgaard and P. Vanhove, "Minimal Basis for Gauge Theory Amplitudes," Phys. Rev. Lett. 103, 161602 (2009) arXiv:0907.1425 [hep-th]].

[35] S. Stieberger, "Open \& Closed vs. Pure Open String Disk Amplitudes," arXiv:0907.2211 [hep-th].

[36] C. R. Mafra, O. Schlotterer and S. Stieberger, "Explicit BCJ Numerators from Pure Spinors," JHEP 1107, 092 (2011) arXiv:1104.5224 [hep-th]].

[37] J. J. Carrasco and H. Johansson, "Five-Point Amplitudes in N=4 SuperYang-Mills Theory and N=8 Supergravity," Phys. Rev. D 85, 025006 (2012) arXiv:1106.4711 [hep-th]].

[38] N. E. J. Bjerrum-Bohr, T. Dennen, R. Monteiro and D. O'Connell, "Integrand Oxidation and One-Loop Colour-Dual Numerators in N=4 Gauge Theory," JHEP 1307, 092 (2013) arXiv:1303.2913 [hep-th]].

[39] S. He, R. Monteiro and O. Schlotterer, "String-inspired BCJ numerators for one-loop MHV amplitudes," arXiv:1507.06288 [hep-th].

[40] R. Monteiro and D. O'Connell, "The Kinematic Algebra From the Self-Dual Sector," JHEP 1107, 007 (2011) doi:10.1007/JHEP07(2011)007 arXiv:1105.2565 [hep-th]].

[41] R. H. Boels, R. S. Isermann, R. Monteiro and D. O'Connell, "ColourKinematics Duality for One-Loop Rational Amplitudes," JHEP 1304, 107 (2013) arXiv:1301.4165 [hep-th]]. 
[42] J. Broedel and L. J. Dixon, "Color-kinematics duality and double-copy construction for amplitudes from higher-dimension operators," JHEP 1210, 091 (2012) arXiv:1208.0876 [hep-th]].

[43] H. Johansson and A. Ochirov, "Color-Kinematics Duality for QCD Amplitudes," arXiv:1507.00332 [hep-ph].

[44] L. de la Cruz, A. Kniss and S. Weinzierl, "Proof of the fundamental BCJ relations for QCD amplitudes," JHEP 1509, 197 (2015) [arXiv:1508.01432 [hep-th]].

[45] J. Nohle, "Color-Kinematics Duality in One-Loop Four-Gluon Amplitudes with Matter," Phys. Rev. D 90, 025020 (2014) arXiv:1309.7416 [hep-th]].

[46] G. Chen and Y. J. Du, "Amplitude Relations in Non-linear Sigma Model," JHEP 1401, 061 (2014) arXiv:1311.1133 [hep-th]].

[47] T. Bargheer, S. He and T. McLoughlin, "New Relations for Three-Dimensional Supersymmetric Scattering Amplitudes," Phys. Rev. Lett. 108, 231601 (2012) doi:10.1103/PhysRevLett.108.231601 arXiv:1203.0562 [hep-th]].

[48] Y. -t. Huang and H. Johansson, "Equivalent D=3 Supergravity Amplitudes from Double Copies of Three-Algebra and Two-Algebra Gauge Theories," Phys. Rev. Lett. 110, 171601 (2013) arXiv:1210.2255 [hep-th]].

[49] Y. -t. Huang, H. Johansson and S. Lee, "On Three-Algebra and Bi-Fundamental Matter Amplitudes and Integrability of Supergravity," JHEP 1311, 050 (2013) arXiv:1307.2222 [hep-th]].

[50] C. R. Mafra and O. Schlotterer, "The Structure of n-Point One-Loop Open Superstring Amplitudes," JHEP 1408, 099 (2014) arXiv:1203.6215 [hep-th]].

[51] J. Broedel, O. Schlotterer and S. Stieberger, "Polylogarithms, Multiple Zeta Values and Superstring Amplitudes," Fortsch. Phys. 61, 812 (2013) arXiv:1304.7267 [hep-th]].

[52] C. R. Mafra and O. Schlotterer, "Multiparticle SYM equations of motion and pure spinor BRST blocks," JHEP 1407, 153 (2014) arXiv:1404.4986 [hep-th]].

[53] C. R. Mafra and O. Schlotterer, "Towards one-loop SYM amplitudes from the pure spinor BRST cohomology," Fortsch. Phys. 63, no. 2, 105 (2015) arXiv:1410.0668 [hep-th]].

[54] C. R. Mafra and O. Schlotterer, "Two-loop five-point amplitudes of super YangMills and supergravity in pure spinor superspace," JHEP 1510, 124 (2015) doi:10.1007/JHEP10(2015)124 arXiv:1505.02746 [hep-th]].

[55] S. He, R. Monteiro and O. Schlotterer, "String-inspired BCJ numerators for oneloop MHV amplitudes," JHEP 1601, 171 (2016) doi:10.1007/JHEP01(2016)171 arXiv:1507.06288 [hep-th]].

[56] N. Arkani-Hamed, F. Cachazo and J. Kaplan, "What is the Simplest Quantum Field Theory?," JHEP 1009, 016 (2010) arXiv:0808.1446 [hep-th]].

[57] J. Broedel and L. J. Dixon, " $\mathrm{R}^{* *} 4$ counterterm and $\mathrm{E}(7)(7)$ symmetry in maximal supergravity," JHEP 1005, 003 (2010) arXiv:0911.5704 [hep-th]]. 
[58] A. Anastasiou, L. Borsten, M. J. Duff, L. J. Hughes and S. Nagy, "A magic pyramid of supergravities," JHEP 1404, 178 (2014) arXiv:1312.6523 [hep-th]].

[59] A. Anastasiou, L. Borsten, M. J. Duff, L. J. Hughes and S. Nagy, "Yang-Mills origin of gravitational symmetries," Phys. Rev. Lett. 113, no. 23, 231606 (2014) arXiv:1408.4434 [hep-th]].

[60] A. Anastasiou, L. Borsten, M. J. Hughes and S. Nagy, "Global symmetries of Yang-Mills squared in various dimensions," JHEP 1601, 148 (2016) arXiv:1502.05359 [hep-th]].

[61] F. Cachazo, S. He and E. Y. Yuan, "Scattering Equations and KLT Orthogonality," arXiv:1306.6575 [hep-th].

[62] F. Cachazo, S. He and E. Y. Yuan, "Scattering of Massless Particles in Arbitrary Dimension," arXiv:1307.2199 [hep-th].

[63] F. Cachazo, S. He and E. Y. Yuan, "Scattering of Massless Particles: Scalars, Gluons and Gravitons," arXiv:1309.0885 [hep-th].

[64] R. Monteiro, D. O'Connell and C. D. White, "Black holes and the double copy," JHEP 1412, 056 (2014) arXiv:1410.0239 [hep-th]].

[65] A. Luna, R. Monteiro, D. O'Connell and C. D. White, "The classical double copy for Taub-NUT spacetime," arXiv:1507.01869 [hep-th].

[66] A. K. Ridgway and M. B. Wise, "Static Spherically Symmetric Kerr-Schild Metrics and Implications for the Classical Double Copy," arXiv:1512.02243 [hepth].

[67] A. Luna, R. Monteiro, I. Nicholson, D. O'Connell and C. D. White, "The double copy: Bremsstrahlung and accelerating black holes," arXiv:1603.05737 [hep-th].

[68] C. D. White, "Exact solutions for the biadjoint scalar field," arXiv:1606.04724 [hep-th].

[69] J. J. M. Carrasco, "Gauge and Gravity Amplitude Relations," arXiv:1506.00974 [hep-th].

[70] M. Gunaydin, S. McReynolds and M. Zagermann, "The R-map and the coupling of $\mathrm{N}=2$ tensor multiplets in 5 and 4 dimensions," JHEP 0601, 168 (2006) hep-th/0511025.

[71] D. Z. Freedman and A. Van Proeyen, "Supergravity," Cambridge University Press (2012).

[72] H. Elvang and Y. t. Huang, "Scattering Amplitudes," arXiv:1308.1697 [hep-th].

[73] Z. Bern, A. De Freitas and H. L. Wong, "On the coupling of gravitons to matter," Phys. Rev. Lett. 84, 3531 (2000) hep-th/9912033.

[74] F. Cachazo, S. He and E. Y. Yuan, "Einstein-Yang-Mills Scattering Amplitudes From Scattering Equations," JHEP 1501 (2015) 121 [arXiv:1409.8256 [hep-th]]. 
[75] F. Cachazo, S. He and E. Y. Yuan, "Scattering Equations and Matrices: From Einstein To Yang-Mills, DBI and NLSM," JHEP 1507, 149 (2015) arXiv:1412.3479 [hep-th]].

[76] E. Casali, Y. Geyer, L. Mason, R. Monteiro and K. A. Roehrig, "New Ambitwistor String Theories," arXiv:1506.08771 [hep-th].

[77] T. Adamo, E. Casali, K. A. Roehrig and D. Skinner, "On tree amplitudes of supersymmetric Einstein-Yang-Mills theory," arXiv:1507.02207 [hep-th].

[78] S. Stieberger and T. R. Taylor, "Graviton as a Pair of Collinear Gauge Bosons," Phys. Lett. B 739 (2014) 457 arXiv:1409.4771 [hep-th]].

[79] S. Stieberger and T. R. Taylor, "Subleading terms in the collinear limit of YangMills amplitudes," Phys. Lett. B 750 (2015) 587 arXiv:1508.01116 [hep-th]].

[80] S. Stieberger and T. R. Taylor, "Graviton Amplitudes from Collinear Limits of Gauge Amplitudes," Phys. Lett. B 744 (2015) 160 arXiv:1502.00655 [hep-th]].

[81] S. Stieberger and T. R. Taylor, "New Relations for Einstein-Yang-Mills Amplitudes," arXiv:1606.09616 [hep-th].

[82] L. F. Alday, J. M. Henn, J. Plefka and T. Schuster, "Scattering into the fifth dimension of N=4 super Yang-Mills," JHEP 1001, 077 (2010) arXiv:0908.0684 [hep-th]].

[83] R. H. Boels, "No triangles on the moduli space of maximally supersymmetric gauge theory," JHEP 1005, 046 (2010) arXiv:1003.2989 [hep-th]].

[84] N. Craig, H. Elvang, M. Kiermaier and T. Slatyer, "Massive amplitudes on the Coulomb branch of N=4 SYM," JHEP 1112, 097 (2011) arXiv:1104.2050 [hep-th]].

[85] S. G. Naculich, "Scattering equations and BCJ relations for gauge and gravitational amplitudes with massive scalar particles," JHEP 1409 (2014) 029 arXiv:1407.7836 [hep-th]].

[86] S. G. Naculich, "CHY representations for gauge theory and gravity amplitudes with up to three massive particles," JHEP 1505, 050 (2015) arXiv:1501.03500 [hep-th]]. 